\title{
The Effectiveness of Various Postpartum Depression Treatments and the Impact of Antidepressant Drugs on Nursing Infants
}

\author{
Dwenda Gjerdingen, MD, MS
}

Background: Postpartum depression is seen in approximately $13 \%$ of women who have recently given birth; unfortunately, it often remains untreated. Important causes for undertreatment of this disorder are providers' and patients' lack of information about the effectiveness of various treatments, and their concerns about the impact of treatment on nursing infants. This article presents research-based evidence on the benefits of various treatments for postpartum depression and their potential risks to nursing infants.

Methods: The medical literature on postpartum depression treatment was reviewed by searching MEDLINE and Current Contents using such key terms as "postpartum depression," "treatment," "therapy," "psychotherapy," and "breastfeeding."

Results and Conclusions: There is evidence that postpartum depression improves with antidepressant drug therapy, estrogen, individual psychotherapy, nurse home visits, and possibly group therapy. of the more frequently studied antidepressant drugs in breastfeeding women, paroxetine, sertraline, and nortriptyline have not been found to have adverse effects on infants. Fluoxetine, however, should be avoided in breastfeeding women. By administering effective treatment to women with postpartum depression, we can positively impact the lives of mothers, their infants, and other family members. ( $\mathrm{J}$ Am Board Fam Pract 2003;16:372-82.)

Postpartum depression, observed in approximately $13 \%$ of women who have recently given birth, ${ }^{1}$ is the most prevalent serious complication of pregnancy. It is classified as a major depressive disorder and as such is characterized by a variety of mental and physical symptoms that produce significant distress and detrimental changes in life functions ( $\mathrm{Ta}-$ ble 1). According to the postpartum onset modifier of major depressive disorder, depressive symptoms begin within the first 4 weeks after delivery; however, it has been shown that women continue to remain at risk for mental disorders even several months after delivery. ${ }^{2}$

Postpartum depression is distinguishable from other postpartum mental disorders. The transient "postpartum blues" occur in a majority of mothers at some time within the first 2 weeks after delivery and are characterized by dysphoria, mood lability, crying, anxiety, insomnia, poor appetite, and irritability. ${ }^{3}$ The more serious but relatively rare post-

Submitted, revised, 18 March 2003.

From the Department of Family Practice \& Community Health, University of Minnesota, St. Paul. Address reprint requests to Dwenda Gjerdingen, MD, 580 Rice St., St. Paul, MN 55103 (e-mail: dgjerdin@umphysicians.umn.edu). partum psychosis (prevalence of $0.1 \%-0.2 \%),{ }^{3}$ is associated with such symptoms as loose thought associations, hallucinations, delusions, and disorganized or catatonic behavior.

Although the consequences of postpartum depression are usually not as severe as those of postpartum psychosis, they can have a significant, negative impact on the lives of not only mothers but also other family members. Mothers themselves might experience physical, marital, parental, social, and vocational difficulties. ${ }^{4}$ Their depression can, in some cases, also adversely affect their infants; studies have noted associations between maternal depression and impaired maternal-infant interactions, ${ }^{5}$ cognitive and emotional development, ${ }^{6}$ and anxiety and lower self-esteem. ${ }^{7}$

Given the potential serious consequences of postpartum depression, it is imperative that health professionals caring for mothers of infants appropriately manage this disorder. A common barrier to providing adequate care is failure to recognize the problem in the first place. Therefore, the US Preventive Services Task Force has recently recommended that adults be screened for depression in clinical practices that have systems in place to as- 
Symptoms

1. Depressed mood most of the day.

2. Markedly diminished interest or pleasure in all, or almost all, activities, most of the day.

3. Marked decrease or increase in appetite, resulting in significant unintentional weight loss or weight gain (ie, $>5 \%$ body weight in 1 month).

4. Insomnia or hypersomnia .

5. Psychomotor agitation or retardation .

6. Fatigue or loss of energy.

7. Feelings of worthlessness or inappropriate guilt.

8. Decreased ability to think or concentrate.

9. Recurrent thoughts of death, or recurrent suicidal thoughts (with or without a plan).

Diagnostic Criteria

Five or more of the symptoms listed above, representing a change in baseline, present nearly every day for the same 2-week period, and producing clinically significant distress or change in functioning

Must include symptom 1 or 2 .

Symptoms do not meet criteria for a mixed episode, and they are not due to drugs, another medical condition, or bereavement (unless prolonged; i.e., $>2$ months)

Adapted from Diagnostic and Statistical Manual of Mental Disorders, 4th ed. Washington, DC: American Psychiatric Association, 1994.

sure accurate diagnosis, effective treatment, and follow-up. ${ }^{8}$ In a study of 342 women, use of the Edinburgh Postnatal Depression Scale (EPDS) to screen for depression at approximately 6 weeks postpartum improved the rate of depression diagnosis from $3.7 \%$ to $10.7 \% .{ }^{9}$ Although the EPDS is the most commonly used screening tool for postpartum depression in research studies, ${ }^{3}$ the Task Force suggests that screening with a simple 2-question tool, developed by Whooley et al (1997), ${ }^{10}$ may be as effective as longer instruments. The tool includes these questions: (1) "Over the past 2 weeks, have you felt down, depressed, or hopeless?" and (2) "Over the past 2 weeks, have you felt little interest or pleasure in doing things?" 10 A positive response to either question indicates a positive screen and should be followed by an expanded history to confirm the diagnosis of depression.

Once the diagnosis of postpartum depression has been established, it should be treated using methods similar to those used for non-postpartum major depressive disorder. These consist of (1) patient education regarding depression, including the biologic basis of depression, treatment options, therapeutic and adverse effects of antidepressant drugs, desired duration of treatment (usually several months or longer), and the need for a healthy lifestyle and social support and (2) the selection of an active treatment modality (usually antidepressant medication and/or psychotherapy) through shared decision making between the patient and provider. ${ }^{11}$ Notably, the treatment of depression in the postpartum period may be more challenging than in other stages of life. Patients and physicians often have concerns about the use of psychotropic medications in breastfeeding women and, related to this, questions about other viable treatment options for breastfeeding women. Therefore, the purpose of this article is to review empirically based information about the effectiveness of various pharmacologic and nonpharmacological treatment modalities for postpartum depression and antidepressant drug effects on nursing infants.

\section{Methods}

A literature search on treatments for postpartum depression (ie, drug therapy, individual and group therapy, and other support therapy) was performed by searching MEDLINE, 1966 to August 2002. The key search terms used were "postpartum depression," "postnatal depression," "puerperal depression," "treatment," "therapy," "drug therapy," "psychotherapy," and "breastfeeding." Current Contents was also searched from 1994 to August 2002 using the key terms "postpartum depression," "puerperal depression," "postnatal depression," "therapy," "psychotherapy," and "group therapy." Searches were mostly limited to randomized controlled trials. Secondary searches were performed using the bibliographies of review articles ${ }^{12-14}$ and other articles obtained through these search meth- 
ods. This procedure yielded 74 articles reporting on 8 types of treatment: antidepressant drugs (4 articles) and their effects on breast milk and nursing infants (50 articles), hormonal treatment (8 articles), individual or group psychotherapy (7 articles), nurse home visits (2 articles), and other treatments, including phototherapy, massage therapy, and electroconvulsive therapy ( 3 articles). Alternative drugs are not discussed here, because information about the safety of these agents is variable. Prophylactic treatments are also not included, because the amount of information on this topic is sufficient for another review.

\section{Results}

\section{Antidepressant Treatment}

Numerous studies have found antidepressant drugs to be effective in treating general depression. ${ }^{15} \mathrm{Al}-$ though newer antidepressants [eg, selective serotonin reuptake inhibitors (SSRIs)] are as efficacious as the older tricyclic antidepressants (TCAs), the SSRIs are the drugs of choice for treating depressive disorders because of their greater tolerability and their relative safety if taken in overdose. ${ }^{15,16}$ Common side effects for TCAs include anticholinergic effects, weight gain, sedation, and orthostatic hypotension, whereas adverse effects for SSRIs include nausea, anorexia, diarrhea, headache, anxiety, nervousness, insomnia, drowsiness, sexual dysfunction, and increased sweating. ${ }^{17}$

Less attention has been given to the efficacy of antidepressant medications for postpartum depression. Uncontrolled studies have typically shown improvement in postpartum depressive symptoms after treatment with antidepressants such as sertraline,${ }^{18}$ fluvoxamine, ${ }^{19}$ and venlafaxine. ${ }^{20}$ However, because depression normally improves over time even without treatment, it is difficult to know whether the improvement in depressive symptoms seen in these studies can be attributed to antidepressant drugs or other factors. A fourth study controlled for such factors by randomizing 87 subjects to 4 groups: fluoxetine or placebo plus 1 or 6 sessions of counseling. Results showed that fluoxetine was better than placebo, and 6 sessions of counseling were better than 1 , but there was no added benefit to combining fluoxetine and multiple counseling sessions. ${ }^{21}$ This trial provides experimental evidence that antidepressant drug therapy is effective in treating postpartum depression.
Antidepressant Treatment in Breastfeeding Mothers

A deterrent to mothers' use of antidepressant medications in the postpartum period is concern about potential adverse effects on the nursing infant. ${ }^{22}$ Several studies have been conducted to inform these concerns. Table 2 reports infant serum levels of antidepressants and behavioral outcomes for breastfeeding newborns whose mothers were treated with a variety of antidepressants, including several SSRIs and TCAs. Infant serum levels of antidepressants, rather than breast milk concentrations, are reported, because serum levels are considered to be more direct determinants of drug exposure. $^{13}$

For most of the infants in these studies, serum levels of antidepressant drugs were either not detectable or very low. Exceptions to this were relatively high infant levels of nefazodone in 1 infant $^{68}$ and fluoxetine in 3 other infants. ${ }^{32,33}$ In each of these cases, disconcerting symptoms were seeneg, increased crying, vomiting, diarrhea, colic, and decreased sleep with fluoxetine, ${ }^{32,33}$ and drowsiness, lethargy, hypothermia, and poor feeding with nefazodone. ${ }^{68}$ The infant whose mother had taken nefazodone was preterm, which may have contributed to the problem. Adverse clinical outcomes were also seen in 1 infant exposed to citalopram, ${ }^{24}$ 2 infants exposed to doxepin, ${ }^{58,60} 1$ infant exposed to nefazodone, ${ }^{68}$ and infants from 3 additional studies with fluoxetine. ${ }^{27,29,31}$ The largest fluoxetine study compared 64 fluoxetine-treated mother-infant pairs with 38 non-treated motherinfant pairs. Statistically significant reductions in infant weight were seen in the fluoxetine group (average deficit, $392 \mathrm{~g}$ between 2 weeks and 6 months of age). ${ }^{29}$ Given the various concerns regarding antidepressant treatment for breastfeeding women, the US Food and Drug Administration has not approved any antidepressant for use during lactation. ${ }^{47}$

These studies provide helpful clinical information about antidepressant transmission to nursing infants, but their methodological weaknesses must also be considered. First, many of the studies listed in Table 2 used a very low sample size; in the majority of reports, only 1 or 2 infants are represented. Second, sampling and measurement methods vary between studies; older studies tend to use less sensitive methods. Third, nursing infants generally ingest relatively small amounts of these drugs-less than $1 \%$ of the maternal dose $;^{71}$ con- 


\begin{tabular}{|c|c|c|c|c|c|}
\hline Study & $\begin{array}{l}\text { No. of } \\
\text { Infants }\end{array}$ & Maternal Dose & $\begin{array}{l}\text { Infant } \\
\text { Age } \\
\text { (weeks) }\end{array}$ & $\begin{array}{l}\text { Infant Serum Drug Levels } \\
\text { (Lower Limit of Detection)* }\end{array}$ & $\begin{array}{l}\text { Adverse Infant-Related } \\
\text { Clinical Outcomes }\end{array}$ \\
\hline \multirow{2}{*}{\multicolumn{6}{|c|}{ Selective Serotonin Reuptake Inhibitors (SSRIs) }} \\
\hline \multicolumn{5}{|c|}{ Citalopram (Celexa) } & \\
\hline Jensen et al, $1997^{23}$ & 1 & $20 \mathrm{mg} / \mathrm{day}$ & 8 & $7 \mathrm{ng} / \mathrm{mL}$ & None \\
\hline Schmidt et al, $2000^{24}$ & 1 & $40 \mathrm{mg} /$ day & 6 & $12.7 \mathrm{ng} / \mathrm{mL}$ & $\begin{array}{l}\text { Uneasy sleep, normalized } \\
\text { with dose reduction }\end{array}$ \\
\hline Rampono et al, $2000^{25}$ & 7 & $\begin{array}{l}0.36 \mathrm{mg} / \mathrm{kg} / \text { day } \\
\text { (median) }\end{array}$ & & $\begin{array}{l}\text { Not detected; } 2.3 \mathrm{ng} / \mathrm{mL} \\
\quad(1 \mathrm{ng} / \mathrm{mL})\end{array}$ & None \\
\hline Spigset et al, $1997^{26}$ & 3 & $20-40 \mathrm{mg} /$ day & $8-16$ & Not discussed & None \\
\hline \multicolumn{6}{|l|}{ Fluoxetine (Prozac) } \\
\hline Brent \& Wisner, $1998^{27}$ & 1 & $20 \mathrm{mg} /$ day & $2-3$ & $61 \mathrm{ng} / \mathrm{mL}$ & $\begin{array}{l}\text { Limp, unresponsive, cyanotic } \\
\text { (mother also taking } \\
\text { carbamazepine and } \\
\text { buspirone) }\end{array}$ \\
\hline Burch \& Wells, $1992^{28}$ & 1 & $20 \mathrm{mg} /$ day & 17 & Not discussed & None \\
\hline Chambers et al, $1999^{29}$ & 64 & Not given & $2-24$ & Not discussed & $\begin{array}{l}\text { Lower growth curves } \\
\quad \text { (average deficit of } 392 \mathrm{~g} \text { ) }\end{array}$ \\
\hline Hendrick et al, $2001^{30}$ & 20 & $10-60 \mathrm{mg} /$ day & $0-31$ & $\begin{array}{l}\text { Fluoxetine, }<1-84 \mathrm{ng} / \mathrm{mL} \text {; } \\
\text { Norfluoxetine, }<1-265 \\
\text { ng/mL }(2 \mathrm{ng} / \mathrm{mL})\end{array}$ & None \\
\hline Isenberg, $1990^{31}$ & 1 & $20 \mathrm{mg} /$ day & 20 & Not discussed & Irritability \\
\hline Kristensen et al, $1999^{32}$ & 14 & $0.24-0.94 \mathrm{mg} / \mathrm{kg} /$ day & $0-60$ & $\begin{array}{l}\text { Not detected; } 252 \mathrm{ng} / \mathrm{mL} \\
\quad(10 \mathrm{ng} / \mathrm{mL})\end{array}$ & $\begin{array}{l}\text { Colic in } 2 \text { infants; irritability } \\
\text { crying, and poor feeding } \\
\text { in } 2 \text { infants (one of these } \\
\text { also had methadone } \\
\text { exposure) }\end{array}$ \\
\hline Lester et al, $1993^{33}$ & 1 & $20 \mathrm{mg} /$ day & 24 & $\begin{array}{l}\text { Fluoxetine, } 340 \mathrm{ng} / \mathrm{mL} ; \\
\quad \text { Norfluoxetine, } 208 \mathrm{ng} / \mathrm{mL}\end{array}$ & $\begin{array}{l}\text { Crying, vomiting, diarrhea, } \\
\text { and decreased sleep, } \\
\text { problem reversed with } \\
\text { formula feeding }\end{array}$ \\
\hline Taddio et al, $1996^{34}$ & 11 & $0.17-0.85 \mathrm{mg} / \mathrm{kg} /$ day & $3-107$ & $\begin{array}{l}\text { Not detected in the one } \\
\text { infant sampled }(1 \mathrm{ng} / \mathrm{mL})\end{array}$ & None \\
\hline Yoshida et al, $1998^{35}$ & 4 & $20-40 \mathrm{mg} /$ day & $4-40$ & $\begin{array}{l}\text { Fluoxetine and nonfluoxetine } \\
\text { not detectable }(2 \mathrm{ng} / \mathrm{mL})\end{array}$ & None \\
\hline \multicolumn{6}{|l|}{ Fluvoxamine (Luvox) } \\
\hline Piontek, $2001^{37}$ & 2 & $300 \mathrm{mg} / \mathrm{day}$ & $2-8$ & Not detected $(2.5 \mathrm{ng} / \mathrm{mL})$ & $\begin{array}{l}\text { None, for up to 2-3 years } \\
\text { after exposure }\end{array}$ \\
\hline Wright et al, $1991^{38}$ & 1 & $200 \mathrm{mg} /$ day & 12 & Not discussed & None \\
\hline Yoshida et al, $1997^{39}$ & 1 & $100-200 \mathrm{mg} /$ day & 17 & Not discussed & None \\
\hline \multicolumn{6}{|l|}{ Paroxetine (Paxil) } \\
\hline Hendrick et al, $2001^{36}$ & 16 & $5-30 \mathrm{mg} / \mathrm{day}$ & $2-26$ & Not detected $(1 \mathrm{ng} / \mathrm{mL})$ & None \\
\hline Misri et al, $2000^{40}$ & 23 & $10-40 \mathrm{mg} /$ day & $4-42$ & Not detected $(0.1 \mathrm{ng} / \mathrm{mL})$ & None \\
\hline Ohman et al, $1999^{41}$ & 7 & $10-40 \mathrm{mg} / \mathrm{day}$ & $6-30$ & Not discussed & None \\
\hline Stowe et al, $2000^{42}$ & 16 & $10-50 \mathrm{mg} /$ day & $4-55$ & Not detected $(2 \mathrm{ng} / \mathrm{mL})$ & None \\
\hline \multicolumn{6}{|l|}{ Sertraline (Zoloft) } \\
\hline Altshuler et al, $1995^{43}$ & 1 & $100 \mathrm{mg} /$ day & $3-7$ & Not detected $(0.5 \mathrm{ng} / \mathrm{mL})$ & None \\
\hline Dodd et al, $2001^{44}$ & 10 & $50-150 \mathrm{mg} /$ day & & Not detected $(2 \mathrm{ng} / \mathrm{mL})$ & None \\
\hline Epperson et al, $2001^{45}$ & 14 & $25-200 \mathrm{mg} / \mathrm{day}$ & $17-26$ & Not detected $(2.5 \mathrm{ng} / \mathrm{mL})$ & None \\
\hline Hendrick et al, $2001^{36}$ & 33 & $25-200 \mathrm{mg} /$ day & $2-60$ & $\begin{array}{l}\text { Not detected in } 28 ; 2-8 \mathrm{ng} / \\
\mathrm{mL} \text { in } 2(1 \mathrm{ng} / \mathrm{mL})\end{array}$ & None \\
\hline Holland, $2000^{46}$ & 6 & Not discussed & $12-16$ & Not discussed & Reduced breast milk supply \\
\hline Stowe et al, $1997^{47}$ & 11 & $25-150 \mathrm{mg} /$ day & $4-141$ & $\begin{array}{l}\text { Sertraline, undetectable or } \\
<3 \mathrm{ng} / \mathrm{mL} \text {; desmethyl- } \\
\text { sertraline, undetectable or } \\
<10 \mathrm{ng} / \mathrm{mL}(1 \mathrm{ng} / \mathrm{mL})\end{array}$ & None \\
\hline Wisner et al, $1998^{48}$ & 9 & $50-200 \mathrm{mg} /$ day & $0-22$ & $\begin{array}{l}\text { Sertraline, not detected or } \\
<64 \mathrm{ng} / \mathrm{mL} ; N \text {-desmethyl- } \\
\text { sertraline, not detected or } \\
<68 \mathrm{ng} / \mathrm{mL}(2 \mathrm{ng} / \mathrm{mL})\end{array}$ & None \\
\hline \multicolumn{6}{|c|}{ Tricyclic Antidepressants (TCAs) } \\
\hline \multicolumn{6}{|l|}{ Amitriptyline (Elavil) } \\
\hline $\begin{array}{l}\text { Bader \& Newman, } \\
\quad 1980^{49}\end{array}$ & 1 & $100 \mathrm{mg} /$ day & 7 & Not detected $(10 \mathrm{ng} / \mathrm{mL})$ & Not discussed \\
\hline $\begin{array}{l}\text { Breyer-Pfaff et al, } \\
1995^{50}\end{array}$ & 1 & $175 \mathrm{mg} /$ day & $0-4$ & Not detected $(5 \mathrm{ng} / \mathrm{mL})$ & None \\
\hline $\begin{array}{l}\text { Brixen-Rasmussen et al, } \\
1982^{51}\end{array}$ & 1 & $75-100 \mathrm{mg} /$ day & $14-30$ & Not detected $(5 \mathrm{ng} / \mathrm{mL})$ & None \\
\hline
\end{tabular}




\begin{tabular}{|c|c|c|c|c|c|}
\hline Study & $\begin{array}{l}\text { No. of } \\
\text { Infants }\end{array}$ & Maternal Dose & $\begin{array}{l}\text { Infant } \\
\text { Age } \\
\text { (weeks) }\end{array}$ & $\begin{array}{l}\text { Infant Serum Drug Levels } \\
\text { (Lower Limit of Detection) }^{*}\end{array}$ & $\begin{array}{l}\text { Adverse Infant-Related } \\
\text { Clinical Outcomes }\end{array}$ \\
\hline Erickson et al, $1979^{52}$ & 1 & $150 \mathrm{mg} /$ day & $8-11$ & Not detected $(28 \mathrm{ng} / \mathrm{mL})$ & Not discussed \\
\hline $\begin{array}{l}\text { Pittard \& O’Neal, } \\
1986^{53}\end{array}$ & 1 & $100 \mathrm{mg} /$ day & $2-3$ & Not discussed & Not discussed \\
\hline Yoshida et al, $1997^{54}$ & 2 & $100-175 \mathrm{mg} /$ day & $4-34$ & $\begin{array}{l}\text { Not detected in one, } 7.5 \mathrm{ng} / \\
\mathrm{mL} \text { in one }(0.1 \mathrm{ng} / \mathrm{mL})\end{array}$ & $\begin{array}{l}\text { Probably none ( } 1 \text { infant was } \\
\text { hypotonic before and after } \\
\text { exposure) }\end{array}$ \\
\hline \multicolumn{6}{|l|}{ Clomipramine (Anafranil) } \\
\hline Schimmell et al, $1991^{55}$ & 1 & $125 \mathrm{mg} /$ day & $1-5$ & $9.8-45.4(20 \mathrm{ng} / \mathrm{mL})$ & None \\
\hline Wisner et al, $1995^{56}$ & 4 & $75-125 \mathrm{mg} /$ day & $2-19$ & $\begin{array}{l}\text { Not detected or not } \\
\text { quantifiable }(10 \mathrm{ng} / \mathrm{mL})\end{array}$ & None \\
\hline Yoshida et al, $1997^{54}$ & 2 & $75-125 \mathrm{mg} /$ day & $20-56$ & $\begin{array}{l}\text { Not detected in one; } 3.2-5.5 \\
\mathrm{ng} / \mathrm{mL} \text { in one }(0.1 \mathrm{ng} / \mathrm{mL})\end{array}$ & None \\
\hline $\begin{array}{l}\text { Desipramine (Norpramin) } \\
\text { Stancer \& Reed } 1986^{57}\end{array}$ & 1 & $300 \mathrm{mg} / \mathrm{dav}$ & $10-11$ & Not detected $(1 \mathrm{ng} / \mathrm{mL})$ & None \\
\hline \multicolumn{6}{|l|}{ Doxepin (Sinequan) } \\
\hline Frey et al, $1999^{58}$ & 1 & $35 \mathrm{mg} /$ day & 1 & $\begin{array}{l}\text { Doxepin, } 10 \mu \mathrm{g} / \mathrm{L} ; N- \\
\text { desmethyldoxepin not } \\
\text { detected }(10 \mathrm{ng} / \mathrm{mL})\end{array}$ & $\begin{array}{l}\text { Poor suckling and } \\
\text { swallowing, drowsiness, } \\
\text { hypotonia, vomiting, } \\
\text { weight loss }\end{array}$ \\
\hline Kemp et al, $1985^{59}$ & 1 & $150 \mathrm{mg} /$ day & 6 & Not detected $(5 \mathrm{ng} / \mathrm{mL})$ & None \\
\hline Matheson et al, $1985^{60}$ & 1 & $75 \mathrm{mg} /$ day & 8 & $\begin{array}{l}\text { Doxepin, } 3 \mu \mathrm{g} / \mathrm{L} ; N- \\
\text { desmethyldoxepin, } 58-66 \\
\mu \mathrm{g} / \mathrm{L}(7 \mathrm{ng} / \mathrm{mL})\end{array}$ & $\begin{array}{l}\text { Sedation, respiratory } \\
\text { depression }\end{array}$ \\
\hline \multicolumn{6}{|l|}{ Imipramine (Tofranil) } \\
\hline Erickson et al, $1979^{52}$ & 1 & $150 \mathrm{mg} /$ day & 8 & Not detected $(28 \mathrm{ng} / \mathrm{mL})$ & Not discussed \\
\hline $\begin{array}{l}\text { Sovner \& Orsulak, } \\
1979^{61}\end{array}$ & 1 & $200 \mathrm{mg} /$ day & 4 & Not discussed & None \\
\hline Yoshida et al, $1997^{54}$ & 4 & $75-150 \mathrm{mg} /$ day & $2-25$ & $\begin{array}{l}\text { Not detected in } 2 ; 0.6-7.4 \\
\mathrm{ng} / \mathrm{mL} \text { in two }(0.1 \mathrm{ng} / \mathrm{mL})\end{array}$ & None \\
\hline \multicolumn{6}{|l|}{ Nortriptyline (Pamelor) } \\
\hline Altshuler et al, $1995^{43}$ & 1 & $125 \mathrm{mg} /$ day & $3-7$ & Not detected $(10 \mathrm{ng} / \mathrm{mL})$ & None \\
\hline Mammen et al, $1997^{62}$ & 2 & Not discussed & $16-31$ & $\begin{array}{l}\text { Nortriptyline not detected; } \\
\text { E-10-hydroxynortriptyline, } \\
<4(2 \mathrm{ng} / \mathrm{mL})\end{array}$ & None \\
\hline $\begin{array}{l}\text { Matheson \& } \\
\quad \text { Skjaeraasen, } 1988^{63}\end{array}$ & 1 & $75-100 \mathrm{mg} /$ day & 1 & Not discussed & None \\
\hline Wisner \& Perel, $1991^{64}$ & 7 & $50-80 \mathrm{mg} /$ day & $0-24$ & $\begin{array}{l}\text { Nortriptyline, not detectable; } \\
\text { 10-hydroxynortriptyline, } \\
5-11 \mathrm{ng} / \mathrm{mL} \text { in } 2 \text { infants } \\
(4-5 \mathrm{ng} / \mathrm{mL})\end{array}$ & None \\
\hline Wisner \& Perel, $1996^{65}$ & 5 & $75-110 \mathrm{mg} /$ day & $4-10$ & Not detected $(<4 \mathrm{ng} / \mathrm{mL})$ & None \\
\hline Wisner et al, $1997^{66}$ & 7 & $60-150 \mathrm{mg} /$ day & $4-6$ & $\begin{array}{l}0-10 \mathrm{ng} / \mathrm{mL} \text { in } 6 \text { term } \\
\text { infants; } 16 \mathrm{ng} / \mathrm{mL} \text { in the } \\
\text { single preterm infant (4 } \\
\mathrm{ng} / \mathrm{mL})\end{array}$ & None \\
\hline \multicolumn{6}{|l|}{ Other Antidepressants } \\
\hline $\begin{array}{l}\text { Buproprion (Wellbutrin) } \\
\text { Briggs et al, } 1993^{67}\end{array}$ & 1 & $100 \mathrm{mg} /$ day & 56 & Not detected $(25 \mathrm{ng} / \mathrm{mL})$ & None \\
\hline $\begin{array}{l}\text { Nefazodone (Serzone) } \\
\text { Yapp et al, } 2000^{68}\end{array}$ & 1 & $300 \mathrm{mg} /$ day & $7-10$ & $\begin{array}{l}1270 \mathrm{ng} / \mathrm{mL} \text { (lower limits of } \\
\text { detection not given) }\end{array}$ & $\begin{array}{l}\text { Drowsiness, lethargy, } \\
\text { hypothermia, and poor } \\
\text { feeding (preterm infant) }\end{array}$ \\
\hline $\begin{array}{l}\text { Venlafaxine (Effexor) } \\
\text { Ilett et al, } 1998^{69}\end{array}$ & 3 & $3-8 \mathrm{mg} / \mathrm{kg} /$ day & $2-24$ & $\begin{array}{l}\text { Venlafaxine, not detected (4 } \\
\text { ng/mL); } O \text {-desmethyl- } \\
\text { venlafaxine, } 23-225 \mathrm{ng} / \mathrm{mL} \\
(3 \mathrm{ng} / \mathrm{mL})\end{array}$ & None \\
\hline Ilett et al, $2002^{70}$ & 7 & $225-300 \mathrm{mg} /$ day & $11-41$ & $\begin{array}{l}\text { Venlafaxine, not detected; } 5 \\
\text { ng/mL; } O \text {-desmethyl- } \\
\text { venlafaxine, } 1.5-5.7 \mathrm{ng} / \mathrm{mL} \\
(1 \mathrm{ng} / \mathrm{mL})\end{array}$ & None \\
\hline
\end{tabular}

*Lower limits of detection are shown in parentheses (in nanograms per milliliter) when the specified drug was not detected in the infant's serum. Lower limits vary with the precision of the laboratory method. 'Safe' infant serum levels of antidepressants have not been established, in that safety profiles depend on the age of the infant (healthy term infants more than 10 weeks old have a lower risk of negative effects), the characteristics of the drug, and concentrations of its metabolites. ${ }^{13}$ 
sequently, their serum levels of antidepressants tend to be very low. However, infants' serum levels of antidepressants might not be representative of their brain levels, because brain tissue is very lipophilic. Fourth, most of the studies had relatively short-term follow-up, lasting weeks to months over the course of antidepressant treatment but not beyond the treatment period. An exception to this is the study by Piontek et $\mathrm{al}^{37}$ of fluvoxamine-exposed infants, where no ill effects were seen after 2 to 3 years of follow-up.

Maternal antidepressant therapy carries risks for nursing infants, but untreated depression is also risky-for mothers and infants. Thus, for each patient, the risks and benefits of treatment must be carefully weighed. If the potential benefits are thought to be greater, paroxetine, sertraline, and nortriptyline could be considered as initial drug therapy options. Each of these medications has been studied in more than 20 mother-infant pairs, with no adverse infant-related events observed. Fluoxetine should be avoided, and citalopram, doxepin, and nefazodone used only cautiously, because adverse effects have been associated with their use. It is generally recommended that treatment be initiated with an SSRI because of ease of administration and low toxicity. ${ }^{14}$ If the patient responds to an initial trial of medication lasting 6 to 8 weeks, the same dose should be continued for at least 6 months after full remission is achieved. ${ }^{14}$

\section{Hormonal Treatment}

Women experience dramatic hormonal shifts with the birth of a child. During pregnancy, levels of endogenous glucocorticoids and estrogens increase, only to plummet after delivery, producing a transient hypoactivation of the hypothalamicpituitary axis that lasts for weeks to months. Cizza et $\mathrm{al}^{72}$ demonstrated that the suppression of the hypothalamic-pituitary axis is more severe and lasts longer in women who develop postpartum blues or depression. In a study of 23 women with severe postpartum depression, 16 had serum estradiol levels below the threshold for gonadal failure. ${ }^{73}$ It follows, then, that the postpartum administration of exogenous hormones might be useful in blunting hormonal and mood declines in women who suffer from postpartum mood disorders.

In 2 prospective observational studies, a benefit to sublingual estrogen treatment of postpartum depression was found after only 2 weeks of treat- ment. ${ }^{73,74}$ These positive outcomes were substantiated in a randomized placebo-controlled trial of 61 depressed mothers. After 1 month, women in the treatment group (200 $\mu \mathrm{g}$ of transdermal $17 \beta$ estradiol administered daily) had fewer depressive symptoms than those in the control group, and this benefit persisted over the 6-month treatment period. ${ }^{75}$ Estrogen may also be effective therapy for postpartum psychosis, according to a few case reports. ${ }^{76,77}$ In contrast, progesterone has not been shown to be beneficial in treating postpartum mood disorders, ${ }^{78}$ and in fact may even be detrimental. ${ }^{79}$

Additional trials should be conducted to confirm these findings and to evaluate the comparative benefit of hormonal versus antidepressant therapy for postpartum depression. Further research is also needed to assess the safety of estrogen treatment in the postpartum period, particularly as it relates to certain risks that are unique to or already increased in the postpartum period, such as decreased milk production in lactating women or thromboemboli.

\section{Individual and Group Psychotherapy}

Another approach to the treatment of postpartum depression is psychotherapy, administered as either an alternative or an adjunct to antidepressant drug therapy. Individual psychotherapy was found to be effective in 4 randomized controlled trials (Table 3). Therapy was administered either by experienced psychotherapists, ${ }^{82}$ trained health visitors, ${ }^{21,81}$ or both. ${ }^{80}$ The number of psychotherapy sessions ranged from 6 to 12. The study by Appleby et $\mathrm{al}^{21}$ was unique in that it compared individual psychotherapy with antidepressant therapy (fluoxetine)both of which, as noted above, were found to be effective. A separate trial showed the value of including the partner in psychotherapy sessions. Women whose partners had participated in several psychotherapy sessions had lower Edinburgh Postnatal Depression Scores at the final assessment (which occurred 10 weeks after the first session) than those whose partners had not participated (8.6 vs 14.7 , respectively). ${ }^{86}$

Group therapy for mothers with postpartum depression has demonstrated mixed results: 2 studies found a benefit, ${ }^{83,85}$ and 1 did not. ${ }^{84}$ These inconsistent outcomes may have resulted from differences in the structure and/or content of the group therapy sessions. For example, in the 2 studies with 
Table 3. Effects of Individual and Group Psychotherapy on Postpartum Depression

\begin{tabular}{|c|c|c|c|}
\hline Study & Study design; $\mathrm{n}$ & Intervention & Outcome \\
\hline \multicolumn{4}{|l|}{ Individual Therapy } \\
\hline Appleby et al, $1997^{21}$ & $\begin{array}{l}\text { Randomized controlled } \\
\text { trial; } \mathrm{n}=87 \text { women } \\
\text { with PPD }\end{array}$ & $\begin{array}{l}4 \text { treatment groups: fluoxetine } \\
\text { or placebo, plus } 1 \text { or } 6 \\
\text { sessions of counseling } \\
\text { provided by trained health } \\
\text { visitors and derived from } \\
\text { cognitive behavioral therapy } \\
\text { (included reassurance and } \\
\text { practical advice about feelings } \\
\text { of not coping, child care, and } \\
\text { lack of enjoyable activities and } \\
\text { practical support) }\end{array}$ & $\begin{array}{l}\text { Six sessions of counseling were } \\
\text { better than } 1 \text { (clinical } \\
\text { interview schedule score } \\
\text { difference }=38.7 \% \text { at } 12 \\
\text { weeks), and fluoxetine was } \\
\text { better than placebo (score } \\
\text { difference }=40.7 \% \text { at } 12 \\
\text { weeks). There was no } \\
\text { advantage in combining } \\
\text { fluoxetine and counseling }\end{array}$ \\
\hline Cooper \& Murray, $1997^{80}$ & $\begin{array}{l}\text { Randomized controlled } \\
\text { trial; } \mathrm{n}=194 \\
\text { primiparous women with } \\
\text { PPD }\end{array}$ & $\begin{array}{l}4 \text { treatment groups: nondirective } \\
\text { counseling }(\mathrm{n}=48), \\
\text { cognitive-behavioral therapy } \\
(\mathrm{n}=42), \text { dynamic } \\
\text { psychotherapy }(\mathrm{n}=48), \text { and a } \\
\text { control group }(\mathrm{n}=54) ; \\
\text { therapy sessions occurred } 1 \\
\text { hour/week for } 10 \text { weeks }\end{array}$ & $\begin{array}{l}\text { Over the initial } 10 \text {-week period, } \\
\text { the } 3 \text { treatment groups } \\
\text { showed greater improvement } \\
\text { than the control group; } \\
\text { however, by } 9 \text { months } \\
\text { postpartum, there was no } \\
\text { significant difference between } \\
\text { groups }\end{array}$ \\
\hline Holden et al, $1989^{81}$ & $\begin{array}{l}\text { Randomized controlled } \\
\text { trial; } \mathrm{n}=55 \text { women } \\
\text { with PPD }\end{array}$ & $\begin{array}{l}8 \text { weekly counseling visits by } \\
\text { health visitors trained to } \\
\text { provide nondirective } \\
\text { counseling (listening to } \\
\text { clients' feelings, and } \\
\text { encouraging them to make } \\
\text { decisions based on their own } \\
\text { judgment) }\end{array}$ & $\begin{array}{l}69 \% \text { of women in the } \\
\text { counseling group versus } 38 \% \\
\text { in the control group had } \\
\text { recovered after } 3 \text { months. }\end{array}$ \\
\hline O'Hara et al, $2000^{82}$ & $\begin{array}{l}\text { Randomized controlled } \\
\text { trial; } \mathrm{n}=120 \text { women } \\
\text { with PPD }\end{array}$ & $\begin{array}{l}12 \text { weekly individual counseling } \\
\text { sessions led by experienced } \\
\text { psychotherapists (discussed } \\
\text { losses \& and interpersonal } \\
\text { conflicts and affirmed clients' } \\
\text { competence) }\end{array}$ & $\begin{array}{l}43.8 \% \text { of women in the } \\
\text { counseling group versus } \\
13.7 \% \text { controls had recovered } \\
\text { after } 3 \text { months. }\end{array}$ \\
\hline \multicolumn{4}{|l|}{ Group Therapy } \\
\hline T1 - : & $\begin{array}{l}\text { Randomized controlled } \\
\text { trial; } \mathrm{n}=60 \text { women } \\
\text { with PPD }\end{array}$ & $\begin{array}{l}4 \text { weekly supportive group } \\
\text { sessions comprising } \\
\text { discussions about transition to } \\
\text { motherhood, postpartum } \\
\text { stress management, } \\
\text { communication skills, life } \\
\text { planning, and strategies for } \\
\text { change }\end{array}$ & $\begin{array}{l}\text { Intervention group members } \\
\text { experienced significant } \\
\text { declines in depression scores, } \\
\text { whereas control group } \\
\text { members did not (Beck } \\
\text { Depression Inventory change: } \\
-6.14 \text { versus }-0.92, P<.01 \text { ). }\end{array}$ \\
\hline Fleming et al, $1992^{84}$ & $\begin{array}{l}\text { Non-randomized } \\
\text { controlled trial; n }=76 \\
\text { depressed \& } 76 \text { non- } \\
\text { depressed mothers }\end{array}$ & $\begin{array}{l}8 \text { weekly unstructured support } \\
\text { groups, facilitated by } \\
\text { psychologists; mothers } \\
\text { discussed childbirth } \\
\text { experiences, mood, } \\
\text { motherhood, changing spousal } \\
\text { relationships, and returning to } \\
\text { work versus staying home }\end{array}$ & $\begin{array}{l}\text { While the entire sample showed } \\
\text { an improvement in mood } \\
\text { from } 2 \text { weeks to } 5 \text { months } \\
\text { postpartum, there was no } \\
\text { significant intervention effect. }\end{array}$ \\
\hline Meager \& Milgrom, $1996^{85}$ & $\begin{array}{l}\text { Randomized controlled } \\
\text { trial; } \mathrm{n}=20 \text { mothers } \\
\text { with PPD }\end{array}$ & $\begin{array}{l}\text { 10-week group treatment } \\
\text { program, included education } \\
\text { (about PPD), cognitive- } \\
\text { behavioral therapy, and } \\
\text { homework for reinforcement }\end{array}$ & $\begin{array}{l}\text { Depression scores dropped } \\
\text { significantly in the } \\
\text { experimental group } \\
\text { (Edinburgh Postnatal } \\
\text { Depression Scale difference: } \\
\text {-9.0), but not in the control } \\
\text { group (difference, } 0.5 \text { ). }\end{array}$ \\
\hline
\end{tabular}

positive outcomes, ${ }^{83,85}$ therapy sessions seemed to be more structured than in the study with negative outcomes (the former 2 studies offered education about such topics as postpartum depression, stress management, communication skills, and life planning, whereas the latter dealt with women's subjec- tive views of childbirth, motherhood, and changing spousal relationships). More research is needed to determine whether group therapy may be helpful for certain populations of depressed mothers and, if so, the manner in which group therapy should be administered. 


\section{Nurse Home Visits}

Another type of postpartum support that has been studied, particularly in Europe, is that provided through nurse or midwife home visits. Two randomized controlled trials have shown a benefit from this type of intervention. In the first, $41 \mathrm{de}-$ pressed mothers were randomized to a control group or a treatment group; treatment group participants received 6 weekly counseling visits by a Child Health Clinic nurse, who acted as a supportive listener. ${ }^{87}$ Compared with the control group, the treatment group experienced a higher rate of recovery from postpartum depression $(80 \%$ vs $25 \%)$. The second trial evaluated the benefit of a nurse home visit program for 181 women with adverse family characteristics. ${ }^{88}$ Visits were made every week for 6 weeks, then every other week for an additional 6 weeks. During their visits, nurses provided guidance on childcare issues, facilitated access to community services, and reinforced successes. Here too, the treatment group showed better outcomes, with Edinburgh Postnatal Depression Scores of 5.7, compared with 7.9 in the control group.

\section{Other Treatments}

In addition to the previously described treatments for postpartum depression, certain physical modalities, such as light therapy, massage therapy, and electroconvulsive therapy, have been tested on a limited basis. Light therapy, often used for seasonal affective disorder, was evaluated in 2 depressed women-one who had been depressed for more than 4 months and refused antidepressant medication and another who been depressed for an unspecified length of time but had not responded to a trial of psychotherapy. ${ }^{89}$ In both women, Hamilton Rating Scale for Depression scores fell markedly after 4 weeks of daily 30-minute phototherapy sessions (from 28 to 29 at baseline to 11 to 12 at follow-up). Field et $\mathrm{al}^{90}$ evaluated massage therapy by randomizing 32 depressed adolescent mothers to relaxation or massage therapy, administered for 30 minutes on 2 consecutive days, for 5 weeks. Results showed that mothers in the massage group had less anxiety, less anxious behavior, and lower stress hormone levels after their sessions than did mothers in the relaxation therapy group. Electroconvulsive therapy, long used for non-postpartum depression, is also thought to be both effective and safe in the postpartum period. ${ }^{91}$ Larger controlled studies on these and other nonpharmaceutical treatments for postpartum depression are needed to expand treatment options, particularly for breastfeeding women.

\section{Summary}

In conclusion, several treatments for postpartum depression have been found to be effective. These include individual psychotherapy and antidepressant drug therapy. Paroxetine, sertraline, and nortriptyline were among the most widely studied antidepressants for which no adverse effects on breastfeeding infants were reported. Other treatments that show promise in managing postpartum depression include estrogen therapy, nurse home visits, and possibly group therapy. More research is needed to identify additional effective treatments, particularly those that are safe for breastfeeding women. These treatments should be combined with patient education about the illness, the specific treatment selected, and other mechanisms for promoting health, such as social support and a healthy lifestyle.

\section{References}

1. O'Hara MW, Swain AM. Rates and risk of postpartum depression-a meta-analysis. Int Rev Psychiatry 1996;8:37-54.

2. Gjerdingen DK, Chaloner KM. The relationship of women's postpartum mental health to employment, childbirth, and social support. J Fam Pract 1994;38: $465-72$.

3. Steiner M. Perinatal mood disorders: position paper. Psychopharmacol Bull 1998;34:301-6.

4. Anonymous. Practice guideline for major depressive disorder in adults. American Psychiatric Association. Am J Psychiatry 1993;150 (4 Suppl):1-26.

5. Murray L, Fiori-Cowley A, Hooper R, Cooper P. The impact of postnatal depression and associated adversity on early mother-infant interactions and later infant outcome. Child Dev 1996;67:2512-26.

6. Beck CT. The effects of postpartum depression on child development: a meta-analysis. Arch Psychiatr Nurs 1998;12:12-20.

7. Politano PM, Stapleton LA, Correll JA. Differences between children of depressed and non-depressed mothers: locus of control, anxiety and self-esteem: a research note. J Child Psychol Psychiatr 1992;33: 451-5.

8. Anonymous. Screening for depression: recommendations and rationale. Am Fam Physician 2002;66: $647-50$.

9. Georgiopoulos AM, Bryan TL, Wollan P, Yawn BP. 
Routine screening for postpartum depression. J Fam Pract 2001;50:117-2.

10. Whooley MA, Avins AL, Miranda J, Browner WS. Case-finding instruments for depression: two questions are as good as many. J Gen Intern Med 1997; $12: 439-45$.

11. Culpepper L. The active management of depression. J Fam Pract 2002;51:769-76.

12. Winans EA. Antidepressant use during lactation. J Hum Lact 2001;17:256-61.

13. Wisner KL, Perel JM, Findling RL. Antidepressant treatment during breast-feeding. Am J Psychiatry 1996;153:1132-37.

14. Wisner KL, Parry BL, Piontek CM. Postpartum depression. N Engl J Med 2002;347:194-9.

15. Mulrow CD, Williams JW, Trivedi M, Chiquette E, Aguilar C, et al. Evidence report on treatment of depression-newer pharmacotherapies. Psychopharmacol Bull 1998;34:409-47.

16. Ables AZ, Baughman OL. Antidepressants: update on new agents and indications. Am Fam Physician 2003;67:547-54.

17. Majeroni BA, Hess A. The pharmacologic treatment of depression. J Am Board Fam Pract 1998;11:12739.

18. Stowe ZN, Casarella J, Landry J, Nemeroff CB. Sertraline in the treatment of women with postpartum major depression. Depression 1995;3:49-55.

19. Suri R, Burt VK, Altshuler LL, Zuckerbrow-Miller J, Fairbanks L. Fluvoxamine for postpartum depression. Am J Psychiatry 2001;158:1739-40.

20. Cohen LS, Viguera AC, Bouffard SM, et al. Venlafaxine in the treatment of postpartum depression. J Clin Psychiatry 2001;62:592-6.

21. Appleby L, Warner R, Whitton A, Faragher B. A controlled study of fluoxetine and cognitive-behavioural counselling in the treatment of postnatal depression. BMJ 1997;314:932-6.

22. Yoshida K, Kumar R. Breast feeding and psychotropic drugs. Int Rev Psychiatry 1996;8:117-24.

23. Jensen PN, Olesen OV, Bertelsen A, Linnet K. Citalopram and desmethylcitalopram concentrations in breast milk and in serum of mother and infant. Ther Drug Monit 1997;19:236-9.

24. Schmidt K, Olesen OV, Jensen PN. Citalopram and breast-feeding: serum concentration and side effects in the infant. Biol Psychiatry 2000;47:164-5.

25. Rampono J, Kristensen JH, Hackett LP, Paech M, Kohan R, Ilett KF. Citalopram and demethylcitalopram in human milk; distribution, excretion and effects in breast fed infants. Br J Clin Pharmacol 2000; 50:263-8.

26. Spigset O, Careborg L, Ohman R, Norstrom A. Excretion of citalopram in breast milk. Br J Clin Pharmacol 1997;44:295-8.

27. Brent NB, Wisner KL. Fluoxetine and carbamaz- epine concentrations in a nursing mother/infant pair. Clin Pediatr (Phila) 1998;37:41-4.

28. Burch KJ, Wells BG. Fluoxetine/norfluoxetine concentration in human milk. Pediatrics 1992;86:676-7.

29. Chambers CD, Anderson PO, Thomas RG, et al. Weight gain in infants breastfed by mothers who take fluoxetine. Pediatrics 1999;104(5):e61.

30. Hendrick V, Stowe ZN, Altshuler LL, et al. Fluoxetine and norfluoxetine concentrations in nursing infants and breast milk. Biol Psychiatry 2001;50: 775-82.

31. Isenberg KE. Excretion of fluoxetine in human breast milk [letter]. J Clin Psychiatry 1990;51:169.

32. Kristensen JH, Ilett KF, Hackett LP, Yapp P, Paech $M$, Begg EJ. Distribution and excretion of fluoxetine and norfluoxetine in human milk. Br J Clin Pharmacol 1999;48:521-7.

33. Lester BM, Cucca J, Andreozzi L, Flanagan P, Oh $W$. Possible association between fluoxetine hydrochloride and colic in an infant. J Am Acad Child Adolesc Psychiatry 1993;32:1253-55.

34. Taddio A, Ito S, Koren G. Excretion of fluoxetine and its metabolite, norfluoxetine, in human breast milk. J Clin Pharmacol 1996;36:42-7.

35. Yoshida K, Smith B, Craggs M, Kumar RC. Fluoxetine in breast-milk and developmental outcome of breast-fed infants. Br J Psychiatry 1998;172: 175-8.

36. Hendrick V, Fukuchi A, Altshuler L, Widawski M, Wertheimer A, Brunhuber MV. Use of sertraline, paroxetine and fluvoxamine by nursing women. Br J Psychiatry 2001;179:163-6.

37. Piontek CM, Wisner KL, Perel JM, Peindl KS. Serum fluvoxamine levels in breastfed infants. J Clin Psychiatry 2001;62:111-3.

38. Wright S, Dawling S, Ashford JJ. Excretion of fluvoxamine in breast milk [letter]. Br J Clin Pharmacol 1991;31:209.

39. Yoshida K, Smith B, Kumar RC. Fluvoxamine in breast-milk and infant development. Br J Clin Pharmacol 1997;44:210-1.

40. Misri S, Kim J, Riggs KW, Kostaras X. Paroxetine levels in postpartum depressed women, breast milk, and infant serum. J Clin Psychiatry 2000;61:828-32.

41. Ohman R, Hagg S, Carleborg L, Spigset O. Excretion of paroxetine into breast milk. J Clin Psychiatry 1999;60:519-23.

42. Stowe ZN, Cohen LS, Hostetter A, Ritchie JC, Owens MJ, Nemeroff CB. Paroxetine in human breast milk and nursing infants. Am J Psychiatry 2000;157:185-9.

43. Altshuler LL, Burt VK, McMullen M, Hendrick V. Breastfeeding and sertraline: a 24-hour analysis. J Clin Psychiatry 1995;56:243-5.

44. Dodd S, Stocky A, Buist A, Burrows GD, Norman TR. Sertraline analysis in the plasma of breast-fed infants. Aust N Z J Psychiatry 2001;35:545-6. 
45. Epperson N, Czarkowski KA, Ward-O'Brien D, et al. Maternal sertraline treatment and serotonin transport in breast-feeding mother-infant pairs. Am J Psychiatry 2001;158:1631-7.

46. Holland D. An observation of the effect of sertraline on breast milk supply [letter]. Aust N Z J Psychiatry 2000;34:1032.

47. Stowe ZN, Owens MJ, Landry JC, et al. Sertraline and desmethylsertraline in human breast milk and nursing infants. Am J Psychiatry 1997;154:1255-60.

48. Wisner KL, Perel JM, Blumer J. Serum sertraline and N-desmethylsertraline levels in breast-feeding mother-infant pairs. Am J Psychiatry 1998;155: 690-2.

49. Bader TF, Newman K. Amitriptyline in human breast milk and the nursing infant's serum. Am J Psychiatry 1980;137:855-6.

50. Breyer-Pfaff U, Nill K, Entenmann KN, Gaertner HJ. Secretion of amitriptyline and metabolites into breast milk. Am J Psychiatry 1995;152:812-3.

51. Brixen-Rasmussen L, Halgrener J, Jorgensen A. Amitriptyline and nortriptyline excretion in human breast milk. Psychopharmacology 1982;76:94-5.

52. Erickson SH, Smith GH, Heidrich F. Tricyclics and breast feeding [letter]. Am J Psychiatry 1979;136: 1483-4.

53. Pittard WB, O’Neal W. Amitriptyline excretion in human milk. J Clin Psychopharmacol 1986;6:383-4.

54. Yoshida K, Smith B, Craggs M, Kumar C. Investigation of pharmacokinetics and of possible adverse effects in infants exposed to tricyclic antidepressants in breast-milk. J Affect Disord 1997;43:225-37.

55. Schimmell MS, Katz EZ, Shaag Y, Pastuszak A, Koren G. Toxic neonatal effects following maternal clomipramine therapy. J Toxicol Clin Toxicol 1991; 29:479-84.

56. Wisner KL, Perel JM, Foglia JP. Serum clomipramine and metabolite levels in four nursing motherinfant pairs. J Clin Psychiatry 1995;56:17-20.

57. Stancer HC, Reed KL. Desipramine and 2-hydroxydesipramine in human breast milk and the nursing infant's serum. Am J Psychiatry 1986;143: 1597-600.

58. Frey OR, Scheidt P, von Brenndorff A. Adverse effects in a newborn infant breast-fed by a mother treated with doxepin. Ann Pharmacother 1999;33: 690-3.

59. Kemp J, Ilett KF, Booth J, Hackett LP. Excretion of doxepin and $\mathrm{N}$-desmethyldoxepin in human milk. Br J Clin Pharmacol 1985;20:497-9.

60. Matheson I, Pande H, Alertsen AR. Respiratory depression caused by $N$-desmethyldoxepin in breast milk [letter]. Lancet 1985;2:1124.

61. Sovner R, Orsulak PJ. Excretion of imipramine and desipramine in human breast milk. Am J Psychiatry 1979;136:451-2.

62. Mammen O, Perel JM, Wheeler S. Antidepressants and breast-feeding. Am J Psychiatry 1997;54: 1174-5.

63. Matheson I, Skjaeraasen J. Milk concentrations of flupenthixol, nortriptyline, and zuclophenthixol and between-breast differences in two patients. Eur J Clin Pharmacol 1988;35:217-20.

64. Wisner KL, Perel JM. Serum nortriptyline levels in nursing mothers and their infants. Am J Psychiatry 1991;148:1234-6.

65. Wisner KL, Perel JM. Nortriptyline treatment of breast-feeding women [letter]. Am J Psychiatry 1996; 153:295.

66. Wisner KL, Perel JM, Findling RL, Hinnes RL. Nortriptyline and its hydroxymetabolites in breastfeeding mothers and newborns. Psychopharmacol Bull 1997;33:249-51.

67. Briggs GG, Samson JH, Ambrose PJ, Schroeder DH. Excretion of bupropion in breast milk. Ann Pharmacother 1993;27:431-3.

68. Yapp P, Ilett KF, Kristensen JH, Hackett LP, Paech MJ, Rampono J. Drowsiness and poor feeding in a breast-fed infant: association with nefazodone and its metabolites. Ann Pharmacother 2000;34:1269-72.

69. Ilett KF, Hackett LP, Dusci LJ, et al. Distribution and excretion of venlafaxine and O-desmethylvenlafaxine in human milk. Br J Clin Pharmacol 1998; 45:459-62.

70. Ilett KF, Kristensen JH, Hackett LP, Paech M, Kohan $\mathrm{R}$, Rampono J. Distribution of venlafaxine and its O-desmethyl metabolite in human milk and their effects in breastfed infants. Br J Clin Pharmacol 2002;53:17-22.

71. Hoffbrand S, Howard L, Crawley H. Antidepressant drug treatment for postnatal depression. Cochrane Database Syst Rev 2001;(2):CD002018.

72. Cizza G, Gold PW, Chrousos GP. High-dose transdermal estrogen, corticotropin-releasing hormone, and postnatal depression [letter]. J Clin Endocrinol Metab 1997;82:704.

73. Ahokas A, Kaukoranta J, Wahlbeck K, Aito M. Estrogen deficiency in severe postpartum depression: successful treatment with sublingual physiologic 17 $\beta$-estradiol: a preliminary study. J Clin Psychiatry 2001;62:332-6.

74. Ahokas AJ, Turtiainen S, Aito M. Sublingual oestrogen treatment of postnatal depression [letter]. Lancet 1998;351:109.

75. Gregoire AJ, Kumar R, Everitt B, Henderson AF, Studd JW. Transdermal oestrogen for treatment of severe postnatal depression. Lancet 1996; 347:930-3.

76. Ahokas A, Aito M. Role of estradiol in puerperal psychosis. Psychopharmacology (Berl) 1999;147: 108-10.

77. Ahokas A, Aito M, Turiainen S. Association between oestradiol and puerperal psychosis. Acta Psychiatr Scand 2000;101:167-70.

78. Granger ACP, Underwood MR. Review of the role 
of progesterone in the management of postnatal mood disorders. J Psychosom Obstet Gynaecol 2001;22:49-55.

79. Lawrie TA, Hofmeyr GJ, DeJager M, Berk M, Paiker J, Viljoen E. A double-blind randomised placebo controlled trial of postnatal norethisterone enanthate: the effect on postnatal depression and serum hormones. Br J Obstet Gynaecol 1998;105:1082-90.

80. Cooper PJ, Murray L. The impact of psychological treatments of postnatal depression on maternal mood and infant development. In: Murray L, Cooper PJ, editors. Postpartum depression and child development. London: Guilford Press, 1997.

81. Holden JM, Sagovsky R, Cox JL. Counselling in a general practice setting: controlled study of health visitor intervention in treatment of postnatal depression. BMJ 1989;298:223-6.

82. O'Hara MW, Stuart S, Gorman LL, Wenzel A. Efficacy of interpersonal psychotherapy for postpartum depression. Arch Gen Psychiatry 2000;57: 1039-45.

83. Chen CH, Tseng YF, Chou FH, Wang SY. Effects of support group intervention in postnatally distressed women: a controlled study in Taiwan. J Psychosom Res 2000;49:395-9.

84. Fleming AS, Klein E, Corter C. The effects of a social support group on depression, maternal attitudes and behavior in new mothers. J Child Psychol Psychiatry 1992;33:685-98.

85. Meager I, Milgrom J. Group treatment for postpartum depression: a pilot study. Aust N Z J Psychiatry 1996;30:852-60.

86. Misri S, Kostaras X, Fox D, Kostaras D. The impact of partner support in the treatment of postpartum depression. Can J Psychiatry 2000;45:554-8.

87. Wickberg B, Hwang CP. Counselling of postnatal depression: a controlled study on a population based Swedish sample. J Affect Disord 1996;39:209-16.

88. Armstrong KL, Fraser JA, Dadds MR, Morris J. A randomized, controlled trial of nurse home visiting to vulnerable families with newborns. J Paediatr Child Health 1999;35:237-4.

89. Corral M, Kuan A, Kostaras D. Bright light therapy's effect on postpartum depression. Am J Psychiatry 2000;157:303-4.

90. Field T, Grizzle N, Scafidi F, Schanberg S. Massage and relaxation therapies' effects on depressed adolescent mothers. Adolescence 1996;31:903-11.

91. Rabheru K. The use of electroconvulsive therapy in special patient populations. Can J Psychiatry 2001; 46:710-9. 